\title{
Contraste de experiencias de estudiantes universitarios en dos escenarios educativos: enseñanza en línea vs. enseñanza remota de emergencia
}

\section{Contrasting the experiences of university students in two educational scenarios: online teaching vs. emergency remote teaching}

\author{
Shamaly Niño Carrasco \\ Universidad Autónoma de Baja California. Mexicali, México \\ shamaly.nino@uabc.edu.mx \\ Juan Carlos Castellanos-Ramírez \\ Universidad Autónoma de Baja California. Mexicali, México \\ juan.castellanos8@uabc.edu.mx \\ Felipe Patrón Espinosa \\ Universidad Autónoma de Baja California. Mexicali, México \\ felipe.patron@uabc.edu.mx
}

\section{RESUMEN}

El objetivo del trabajo fue explorar las experiencias de estudiantes universitarios sobre la acción instruccional recibida en un escenario de enseñanza en línea, antes de la Covid-19, y en un escenario de enseñanza remota de emergencia, durante la pandemia. Se realizó un estudio transversal-correlacional con muestreo no probabilístico de participantes voluntarios; la muestra estuvo conformada por 301 estudiantes matriculados en una universidad mexicana. Para contrastar las experiencias de los estudiantes en los dos escenarios, se empleó un cuestionario autoaplicado de escala tipo Likert de 24 ítems (coeficiente de consistencia interna Alfa de Cronbach de .968 , correlaciones mayores a .30 en todos los ítems); el procedimiento de análisis comportó dos vías complementarias: el cálculo de frecuencias porcentuales y la aplicación de la prueba t de Student para muestras relacionadas. Se constató que las medias fueron mayores para el escenario de enseñanza en línea en comparación con el escenario de enseñanza remota de emergencia, aunque solo se encontraron diferencias estadísticamente significativas en torno al diseño instruccional, la evaluación de los aprendizajes y la participación de los estudiantes en las asignaturas. Los resultados evidencian un par de limitaciones metodológicas y, a la vez, un conjunto de preguntas que abren líneas futuras de trabajo.

Palabras clave: Covid-19, cuestionario, educación superior, enseñanza en línea, enseñanza remota de emergencia. 


\begin{abstract}
The objective of these work was to explore the experiences of university students about the instructional action in an online teaching setting, before Covid-19, and in emergency remote teaching setting, during the pandemic. For this, a cross sectionalcorrelational study with non-probabilistic sampling of voluntary participants was carried out; the sample consisted of 301 students enrolled in a Mexican university. To contrast the experiences of the students in the two scenarios, a self-applied Likerttype questionnaire was used, composed of 24 items (Cronbach's alpha internal consistency coefficient of .968, correlations greater than .30 in all items); the analysis procedure involved two complementary routes: calculating percentage frequencies and in addition Student's $t$ test was applied for related samples. It was found that the means were higher for the online teaching scenario compared to the emergency remote teaching scenario, although statistically significant differences were only found regarding the instructional design, the evaluation of learning and the participation of students in the subjects. The results also reveal a couple of methodological limitations and, at the same time, a set of questions that open future lines of work.
\end{abstract}

Keywords: Covid-19, emergency remote teaching, higher education, online teaching, questionnaire.

\title{
INTRODUCCIÓN
}

En las últimas dos décadas las catástrofes naturales se han incrementado considerablemente en distintas partes del mundo provocando, además de pérdidas humanas, fuertes desequilibrios económicos y sociales en los países (Esnard et al., 2018; Mutch, 2017). De manera reciente, los desastres producidos por epidemias virales han representado un intermitente problema de salud pública a nivel global derivado, en gran medida, de la gran movilización de personas a través de los continentes (Bloom y Cadarette, 2019; Quinn y Kumar, 2014).

Ante el brote de una epidemia, las medidas de distanciamiento social son esenciales para reducir los índices de contagio en la población (Ahmed et al., 2018; Huremovic, 2019). Como parte de las estrategias de distanciamiento social, la Organización Mundial de la Salud -OMS- (2009) recomienda el cierre de espacios públicos, entre ellos los centros educativos. Al respecto, diversas investigaciones constatan que el cierre de escuelas en una etapa temprana de la epidemia contribuye potencialmente a controlar la transmisión comunitaria y ayuda indirectamente a reducir la carga viral (Brown et al., 2011; Carlo y Chung, 2009; Halder et al., 2010).

De acuerdo con la OMS (2009) se estima que el cierre de escuelas reduce entre un $30 \%$ y $50 \%$ la demanda de asistencia sanitaria en el pico de una pandemia, sin embargo, dicha medida puede tener efectos colaterales y negativos en el rendimiento académico de los estudiantes, principalmente en aquellos que provienen de familias pobres, que sufren alguna discapacidad o que se ubican en zonas marginadas (Buerger y Harris, 2015; Daniel, 2020; Satpathy y Ali, 2020). Como refieren Viner et al. (2020), la educación es

Contraste de experiencias de estudiantes universitarios en dos escenarios educativos: enseñanza en línea vs. enseñanza remota de emergencia. Niño, Castellanos-Ramírez y Patrón. 
uno de los servicios públicos de mayor importancia para el desarrollo social y crecimiento económico de los países, por lo que un cierre prolongado puede provocar, en el futuro, una regresión en la productividad nacional.

Experiencias previas han demostrado que, ante brotes de enfermedades infecciosas, el uso de herramientas tecnológicas permite a los estudiantes continuar sus estudios desde casa, sin tener que poner en riesgo su bienestar físico y desarrollo cognitivo (Murphy, 2020); de hecho, los antecedentes más inmediatos remiten a los esfuerzos de las escuelas de Beijing, Hong Kong, Singapur y Toronto al trasladar sus programas educativos presenciales a la modalidad en línea ante el brote del virus SARS-CoV en el año 2003 (Chan, 2005; Fox, 2004; Pang et al., 2003; Rhea, 2003). De manera similar, en el año 2009, diversas universidades de Estados Unidos de América habilitaron plataformas tecnológicas para que los estudiantes continuaran sus estudios profesionales durante el brote de influenza H1N1 (Meyer y Wilson, 2011).

La reciente pandemia provocada por el virus SARS-CoV-2 y sus repercusiones en el ámbito educativo no tiene precedentes en la historia de la humanidad. El 24 de marzo de 2020, la Organización de las Naciones Unidas para la Educación, la Ciencia y la Cultura (UNESCO, por sus siglas en inglés) estimó que 138 países habían implementado cierres de escuelas a nivel nacional afectando, de esta manera, la formación de 1,370 millones de niños y jóvenes, aproximadamente el $80 \%$ de la población estudiantil mundial (UNESCO, 2020a). En México, más de 37 millones de estudiantes se vieron afectados por el cierre de 265 mil centros educativos, de los cuales aproximadamente cuatro millones de estudiantes pertenecen al nivel superior (UNESCO, 2020b).

Ante la emergencia sanitaria por la Covid-19, en menos de una semana, las universidades mexicanas trasladaron sus programas educativos presenciales a la modalidad en línea utilizando los recursos tecnológicos disponibles y a su alcance para dar continuidad al ciclo escolar (Amaya et al., 2021; Gazca, 2020; Sánchez et al., 2020). Al mismo tiempo que se tomaban estas medidas, se propagó una gran cantidad de información en torno a la utilización de recursos tecnológicos para la educación en línea, tanto de carácter comercial como periodístico (De la Calle et al., 2021). Mientras que los medios de comunicación utilizaron términos como "educación en línea" y "escolarización en el hogar", los especialistas en tecnología educativa propusieron el término de Enseñanza remota de emergencia (Bozkurt y Sharma, 2020; Glenn, 2020; Hodges et al., 2020; Innes, 2020) para referirse a una adaptación forzada de programas educativos presenciales y su habilitación improvisada dentro de plataformas tecnológicas.

Como Bozkurt y Sharma (2020) señalan:

In appearance, we are currently engaged in seems like online distance education, however, in essence, this is rather a temporary solution, one that would be more properly named emergency remote teaching. In other words, online distance education is one thing and emergency remote teaching is another thing (p.2).

En efecto, más allá de la utilización de recursos tecnológicos para la continuidad académica durante el confinamiento, el éxito de la educación en línea se basa fundamentalmente en tres principios básicos: i) la calidad de sus diseños instruccionales finamente pautados para orientar a los estudiantes en sus procesos de construcción del

Contraste de experiencias de estudiantes universitarios en dos escenarios educativos: enseñanza en línea vs. enseñanza remota de emergencia. Niño, Castellanos-Ramírez y Patrón. 
conocimiento (Gomes et al., 2021; Park y Luo, 2017; Rozitis, 2017), ii) el alto nivel de especialización que los profesores tienen sobre la utilización de los recursos tecnológicos para el aprendizaje y la formación pedagógica para proporcionar andamios cognitivos a sus estudiantes (Compton, 2009; Comas et al., 2012; Watson et al., 2017) y iii) sus esquemas de evaluación para valorar los aprendizajes de manera continua y brindar retroalimentación oportuna durante el proceso formativo para la mejora del desempeño grupal e individual (Alvarado, 2014; Shintani, 2016).

Dicho lo anterior, en un escenario de emergencia donde se requieren acciones rápidas y contingentes por parte de las instituciones educativas, cumplir con los principios fundamentales básicos de la educación en línea resulta casi imposible de lograr (Álvarez, 2020; Cervantes y Gutiérrez, 2020; Fernández et al., 2020). Por lo tanto, el objetivo de la enseñanza remota de emergencia, más que recrear un sistema de formación en línea en medio de la crisis, es suavizar la brecha de aprendizaje entre los estudiantes a través de la utilización de recursos tecnológicos, mientras se resuelve la incidencia (Houston, 2017; Meyer y Wilson, 2011).

Durante la emergencia educativa por la Covid-19, una de las limitaciones que los profesores han enfrentado es, justamente, la falta de experiencia y escasa formación en la utilización de plataformas tecnológicas y facilitación del aprendizaje en línea (Bozkurt y Sharma, 2020; Glenn, 2020; Muller y Goldenber, 2020). Diversas investigaciones (Bolliger y Wasilik, 2009; Booth et al., 2017; Fernández et al., 2020) coinciden al señalar que cuando los profesores incursionan por primera vez en ambientes educativos en línea experimentan una sensación de agobio y dificultad para impartir sus clases, sobre todo, por la carga de trabajo y tiempo que invierten para planificar actividades, resolver dudas, revisar trabajos y retroalimentar a sus estudiantes. De hecho, se estima que "an instructor requires 10 hours to design and develop one hour of online instruction. This estimate does not include the hours instructors spend on faculty training and development" (Bolliger y Wasilik, 2009, p.14.).

Aunque algunos centros educativos implementaron cursos de formación docente sobre la marcha para solventar esas deficiencias formativas (Rajab et al., 2020; Subedi et al., 2020), no hubo tiempo suficiente para que los profesores interiorizaran y transfirieran dicho conocimiento a sus prácticas de enseñanza remota (García et al., 2020), por lo que probablemente estas experiencias educativas -tanto de profesores como de estudiantessean menos satisfactorias que aquellas de los programas educativos expresamente diseñados para su impartición línea.

En este marco, el propósito del presente trabajo fue explorar, en una misma población de estudiantes universitarios, sus experiencias y valoraciones en torno a la instrucción recibida por parte de sus profesores, en dos escenarios educativos distintos: i) un escenario de enseñanza en línea, que remite a las asignaturas en línea previamente cursadas por los estudiantes y diseñadas expresamente para operarse en la modalidad virtual antes de la emergencia por la Covid-19; y ii) un escenario de enseñanza remota de emergencia, que refiere a las asignaturas presenciales que fueron habilitadas de manera espontánea para su impartición en línea durante el período de confinamiento.

Contraste de experiencias de estudiantes universitarios en dos escenarios educativos: enseñanza en línea vs. enseñanza remota de emergencia. Niño, Castellanos-Ramírez y Patrón. 


\section{MÉTODO}

\section{Participantes}

El estudio se caracterizó por ser transversal-correlacional con muestreo no probabilístico de participantes voluntarios (Hernández et al., 2014) con el fin de indagar, desde el punto de vista de los estudiantes, sobre las prácticas de enseñanza en línea antes de la emergencia por Covid-19 y su contraste con las prácticas de enseñanza remota durante la emergencia. La muestra estuvo conformada por 301 estudiantes inscritos en los diferentes programas educativos de licenciatura ofertados por la Facultad de Ciencias Humanas, en la Universidad Autónoma de Baja California. El rango de edad de los estudiantes osciló entre 19 y 24 años, de los que el 62\% eran mujeres y el 38\% eran hombres.

Una condición común entre los estudiantes fue su experiencia previa en el aprendizaje en línea, es decir, todos los participantes del estudio habían cursado dos o más asignaturas en línea dentro de sus programas educativos antes de la emergencia por Covid-19; esta condición fue importante, ya que permitió hacer el contraste entre las experiencias de los estudiantes en la realización de cursos en línea y las experiencias obtenidas en el escenario de enseñanza remota de emergencia.

\section{Instrumento}

Para conocer las experiencias y valoraciones de los estudiantes en torno a los escenarios de enseñanza en línea y enseñanza remota de emergencia se empleó la primera versión del Cuestionario de Satisfacción Instruccional (CSI) elaborado por Niño, Patrón y Castellanos-Ramírez (2020). El CSI es un instrumento autoaplicado de escala tipo Likert compuesto de 24 ítems que, en su primera versión, obtuvo un coeficiente de consistencia interna Alfa de Cronbach de .968, con correlaciones mayores a .30 en todos los ítems.

El cuestionario consta de cuatro categorías, cada una con seis ítems:

1. Categoría de experiencias en torno al diseño de la actividad: ítems relativos a i) la organización de actividades, ii) tiempo para la realización de tareas, iii) estrategias y recursos didácticos programados, iv) relevancia de los contenidos proyectados, v) complejidad gradual de las tareas propuestas y vi) volumen razonable de tareas planificadas.

2. Categoría de experiencias en torno a la instrucción directa: ítems relativos a i) claridad y precisión de las instrucciones, ii) seguimiento de actividades, iii) flexibilidad académica, iv) diversificación de canales comunicación, v) inmediatez en las respuestas del profesor para resolver dudas y vi) clima de confianza.

3. Categoría de experiencias en torno a la evaluación de los aprendizajes: ítems respecto a i) claridad en criterios generales para la evaluación del curso, ii) claridad en criterios específicos (o rúbricas) para la evaluación de tareas, iii) retroalimentación por parte del profesor sobre las tareas elaboradas, iv) retroalimentación de tareas en un tiempo razonable y antes de dar inicio a la

Contraste de experiencias de estudiantes universitarios en dos escenarios educativos: enseñanza en línea vs. enseñanza remota de emergencia. Niño, Castellanos-Ramírez y Patrón. 
siguiente actividad, v) retroalimentación clara y útil para mejorar la realización de trabajos posteriores y vi) estimaciones adecuadas para asignar valor a las tareas según la dificultad y tiempo invertido en ellas.

4. Categoría de experiencias sobre la participación en asignaturas: ítems relacionados con i) nivel de desempeño propio, como participación, asistencia, puntualidad y cumplimiento de las tareas, ii) desempeño de los profesores, iii) diseño de las actividades, tipos de tareas y formas de trabajo, iv) carga de trabajo y nivel de exigencia, v) evaluación de los aprendizajes y vi) valoración global de la experiencia.

Los mismos ítems y categorías se utilizaron para conocer las experiencias y valoraciones de los estudiantes en torno a los dos escenarios educativos (enseñanza en línea y enseñanza remota de emergencia); solamente se realizaron ajustes en la conjugación verbal de los ítems para adecuarlos a cada escenario.

Las opciones de respuesta a los ítems se puntuaron con valores del 1 al 5. Es importante señalar que, en el caso de las primeras tres categorías del cuestionario (diseño, instrucción y evaluación) en donde los ítems se relacionan con las experiencias de los estudiantes, las respuestas se formularon en una escala de frecuencia (5=Siempre, 4=Casi siempre, $3=$ Algunas veces, $2=$ Casi nunca y $5=$ Nunca). En cambio, en la cuarta categoría del cuestionario relacionada con las experiencias de los estudiantes sobre su participación en las asignaturas, las respuestas a los ítems se formularon en una escala de satisfacción (5=Muy buena, 4=Buena, 3=Regular, Mala=2, Muy mala=1). La suma de la opción elegida en cada uno de los ítems se tomó como la calificación general para cada una de las cuatro categorías. Cabe destacar que las calificaciones altas indican experiencias y valoraciones más positivas.

\section{Procedimiento}

Dos investigadores con experiencia previa en el estudio de procesos educativos mediados por tecnología se reunieron para discutir los rasgos característicos de la educación en línea y formular, a la luz de la teoría, los ítems del cuestionario. El diseño de la primera versión del instrumento se sometió a evaluación de jueces expertos quienes valoraron la coherencia, relevancia, claridad y suficiencia de los ítems. A partir de las observaciones realizadas por los jueces, se elaboró una segunda versión del instrumento que fue piloteada con una muestra inicial de 20 estudiantes; como resultado del pilotaje se hicieron modificaciones adicionales para mejorar la comprensión de algunos ítems.

Finalmente el cuestionario se habilitó de manera digital en la plataforma Qualtrics y su difusión se realizó a través de las páginas oficiales de la institución educativa. Todos los participantes fueron informados del objetivo del estudio, por lo que su participación fue voluntaria. Cabe mencionar que el cuestionario se cumplimentó al cierre del periodo escolar, antes de que los estudiantes conocieran su calificación final.

Contraste de experiencias de estudiantes universitarios en dos escenarios educativos: enseñanza en línea vs. enseñanza remota de emergencia. Niño, Castellanos-Ramírez y Patrón. 


\section{Análisis de datos}

Debido a que el CSI permite comparar la acción instruccional de los profesores en dos escenarios distintos para cada una de las categorías (diseño, instrucción, evaluación, participación) se calcularon, primero, las frecuencias porcentuales de cada escala y, después, se aplicó la prueba t de Student para muestras relacionadas; en este último caso, para todas las pruebas $\alpha$ fue igual a 0.05 . El análisis estadístico se realizó a través del software IBM SPSS Statistics 23 y el tamaño del efecto y la potencia estadística para cada prueba se calculó por medio del software G*Power 3.1.

Como se aprecia, el procedimiento de análisis incluyó dos vías complementarias. La primera, basada en el reporte de las frecuencias porcentuales, permitió identificar el escenario y los elementos instruccionales que, desde la perspectiva de los estudiantes, resultan de forma más positiva sobre su proceso formativo. La segunda, orientada por los resultados de la primera vía, consistió en aplicar la prueba t de Student para confirmar la presencia de diferencias estadísticamente significativas en las categorías analizadas en cada escenario.

En suma, las dos vías de análisis complementarias permiten contrastar las experiencias de los estudiantes sobre su proceso formativo en ambos escenarios, no con la intención de emitir juicios valorativos sobre la calidad del aprendizaje, sino con el fin de reconocer los elementos instruccionales que resultan más favorables para los estudiantes y que podrían considerarse para mejorar la acción instruccional desplegada tanto en el escenario de enseñanza en línea como en el escenario de enseñanza remota de emergencia.

\section{RESULTADOS}

En la Tabla 1 se muestran los porcentajes relativos a las respuestas proporcionadas por los estudiantes sobre las experiencias con el diseño de actividades en los escenarios de enseñanza en línea (EL) y enseñanza remota de emergencia (ER). En las columnas de los rangos considerados como más favorables (siempre y casi siempre) se distinguen los valores iguales o superiores (celdas con datos en negritas) de los valores inferiores (celdas con datos sin negritas) a un porcentaje equivalente al 35\%; en las columnas de los rangos considerados como menos favorables (casi nunca y nunca) se distinguen los valores iguales o superiores (celdas con datos en negritas) de los valores inferiores (celdas con datos sin negritas) a un porcentaje equivalente al $10 \%$.

Contraste de experiencias de estudiantes universitarios en dos escenarios educativos: enseñanza en línea vs. enseñanza remota de emergencia. Niño, Castellanos-Ramírez y Patrón. 
RED. Revista de Educación a Distancia. Núm. 65, Vol. 21. Artíc. 7, 08-01-2021

DOI: https://doi.org/10.6018/red.440731

Tabla 1

Experiencias de los estudiantes en torno al diseño de las actividades

\begin{tabular}{|c|c|c|c|c|c|c|c|c|c|c|}
\hline \multirow[b]{2}{*}{ Ítems } & \multicolumn{2}{|c|}{ Siempre } & \multicolumn{2}{|c|}{ Casi siempre } & \multicolumn{2}{|c|}{ Algunas veces } & \multicolumn{2}{|c|}{ Casi nunca } & \multicolumn{2}{|c|}{ Nunca } \\
\hline & $\begin{array}{c}\text { EL } \\
\% \\
\end{array}$ & $\begin{array}{c}\mathrm{ER} \\
\% \\
\end{array}$ & $\begin{array}{c}\mathrm{EL} \\
\% \\
\end{array}$ & $\begin{array}{c}\mathrm{ER} \\
\%\end{array}$ & $\begin{array}{c}\mathrm{EL} \\
\% \\
\end{array}$ & $\begin{array}{c}\mathrm{ER} \\
\% \\
\end{array}$ & $\begin{array}{l}\text { EL } \\
\% \\
\end{array}$ & $\begin{array}{c}\mathrm{ER} \\
\% \\
\end{array}$ & $\begin{array}{l}\mathrm{EL} \\
\% \\
\end{array}$ & $\begin{array}{c}\mathrm{ER} \\
\%\end{array}$ \\
\hline $\begin{array}{l}\text { Organización } \\
\text { favorable de } \\
\text { actividades }\end{array}$ & 29,19 & 20,33 & 40,91 & 32,54 & 22,25 & 29,90 & 5,50 & 13,16 & 2,15 & 4,07 \\
\hline $\begin{array}{l}\text { Asignación de } \\
\text { tiempo suficiente } \\
\text { para la realización } \\
\text { de actividades }\end{array}$ & 39,23 & 27,27 & 37,56 & 30,62 & 18,18 & 30,86 & 3,59 & 8,85 & 1,44 & 2,39 \\
\hline $\begin{array}{l}\text { Diversidad de } \\
\text { estrategias y } \\
\text { recursos didácticos }\end{array}$ & 25,84 & 16,51 & 38,28 & 29,90 & 27,03 & 33,25 & 6,94 & 15,55 & 1,91 & 4,78 \\
\hline $\begin{array}{l}\text { Relevancia de los } \\
\text { contenidos }\end{array}$ & 23,44 & 19,62 & 34,69 & 31,58 & 30,38 & 28,71 & 8,13 & 15,55 & 3,35 & 4,55 \\
\hline $\begin{array}{l}\text { Complejidad } \\
\text { progresiva de las } \\
\text { tareas }\end{array}$ & 31,34 & 28,95 & 38,52 & 32,78 & 23,44 & 28,23 & 5,26 & 8,13 & 1,44 & 1,91 \\
\hline $\begin{array}{l}\text { Volumen razonable } \\
\text { de tareas }\end{array}$ & 4,55 & 1,91 & 18,90 & 10,77 & 37,08 & 26,08 & 25,12 & 34,69 & 14,35 & 26,56 \\
\hline
\end{tabular}

Nota. EL = Enseñanza en línea, ER = Enseñanza remota de emergencia

En relación con el diseño de las asignaturas en línea cursadas antes de la emergencia por la Covid-19 (EL), los cuatro aspectos que los estudiantes consideran más favorables sobre el diseño de las actividades son la organización (40,91\%), la asignación de tiempo suficiente para la realización de actividades $(39,23 \%)$, la complejidad progresiva de las tareas $(38,52 \%)$ y la diversidad de estrategias y recursos didácticos $(38,28 \%)$; en cambio, el aspecto considerado como menos favorable es el volumen de tareas $(25,12 \%)$.

En las valoraciones de los estudiantes sobre el diseño de actividades cursadas en el escenario de enseñanza remota de emergencia (ER) no se observan valores superiores al $35 \%$ en los rangos considerados como más favorables (siempre y casi siempre); en cambio, los cuatro aspectos que los estudiantes consideran como menos favorables (casi nunca y nunca) son el volumen de las tareas $(34,69 \%)$, la diversidad de las estrategias y recursos didácticos $(15,55 \%)$, la relevancia de los contenidos $(15,55 \%)$ y la organización de las actividades $(13,16 \%)$.

A efectos de visualizar el contraste entre las experiencias de los estudiantes sobre el diseño de actividades en los escenarios EL y ER, en la Figura 1 se presenta, de manera global, la suma de los dos porcentajes más altos obtenidos en cada ítem; en las barras se distinguen los porcentajes de los rangos siempre (entramado gris fuerte), casi siempre (entramado gris claro), algunas veces (entramado con rayas), casi nunca (entramado con puntos) y nunca (sin entramado).

Contraste de experiencias de estudiantes universitarios en dos escenarios educativos: enseñanza en línea vs. enseñanza remota de emergencia. Niño, Castellanos-Ramírez y Patrón. 


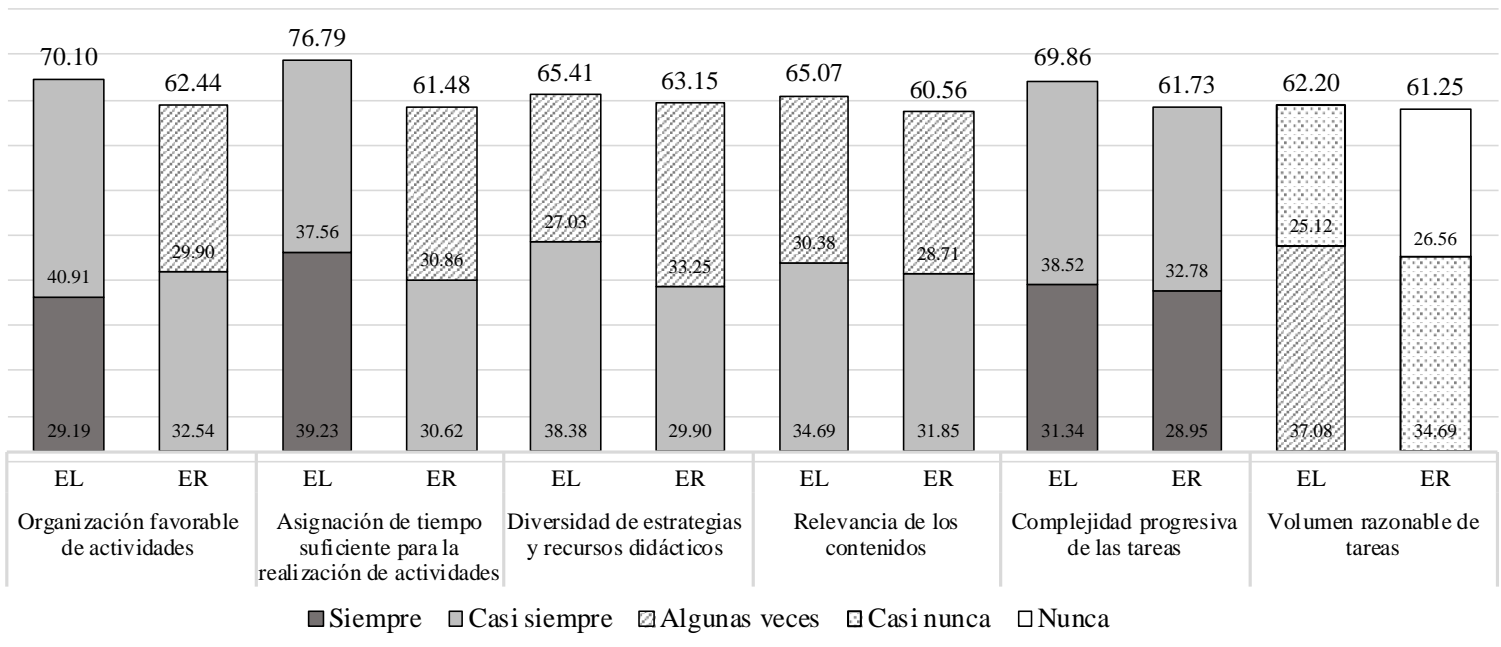

Figura 1. Contraste de las experiencias de los estudiantes sobre el diseño de las actividades en los escenarios de enseñanza en línea (EL) y enseñanza remota de emergencia (ER).

De acuerdo con la Figura 1, los aspectos relacionados con el diseño de actividades que los estudiantes consideran más favorables son los que se llevan a cabo en el escenario EL, en concreto, la asignación de tiempo para la realización de actividades $(76,79 \%)$ y su organización $(70,10 \%$ ), ya que no solo destacan por presentar porcentajes arriba del $70 \%$, sino que además son el resultado de sumar porcentajes de los rangos siempre y casi siempre. En contraste, pese a que el escenario ER presenta porcentajes arriba del $60 \%$ en ambos aspectos, estos son el resultado de sumar porcentajes de los rangos casi siempre y algunas veces.

Aun cuando en el resto de los ítems no se observan diferencias contundentes entre los porcentajes totales y el tipo de rangos de cada escenario, sí se distinguen diferencias en los porcentajes de cada rango; en detalle, la diversidad de estrategias y recursos didácticos es más o menos favorable para el 65,41\% de los estudiantes en el escenario EL y para el $63,15 \%$ en el escenario ER, sin embargo, el escenario EL destaca porque el 38,38\% corresponde al rango casi siempre, mientras que en el escenario ER el porcentaje más alto $(33,25 \%)$ se concentra en el rango algunas veces. De manera similar, el volumen de tareas es considerado como poco favorable para el $62,20 \%$ de los estudiantes en el escenario EL y para el $61,25 \%$ en el escenario ER; no obstante, nuevamente destaca el escenario EL, ya que el 37,08\% corresponde al rango algunas veces, mientras que en el escenario ER el porcentaje más alto $(34,69 \%)$ se concentra en el rango casi nunca y, además, porque el $26,56 \%$ restante corresponde al rango nunca.

La Tabla 2 muestra los porcentajes correspondientes a las respuestas de los estudiantes en torno a la instrucción directa en los escenarios EL y ER; en esta se conserva el formato de presentación de la tabla anterior.

Contraste de experiencias de estudiantes universitarios en dos escenarios educativos: enseñanza en línea vs. enseñanza remota de emergencia. Niño, Castellanos-Ramírez y Patrón. 
RED. Revista de Educación a Distancia. Núm. 65, Vol. 21. Artíc. 7, 08-01-2021

DOI: https://doi.org/10.6018/red.440731

Tabla 2

Experiencias de los estudiantes en torno a la instrucción directa

\begin{tabular}{|c|c|c|c|c|c|c|c|c|c|c|}
\hline \multirow{2}{*}{ Ítems } & \multicolumn{2}{|c|}{ Siempre } & \multicolumn{2}{|c|}{ Casi siempre } & \multicolumn{2}{|c|}{$\begin{array}{c}\text { Algunas } \\
\text { veces }\end{array}$} & \multicolumn{2}{|c|}{ Casi nunca } & \multicolumn{2}{|c|}{ Nunca } \\
\hline & $\begin{array}{c}\text { EL } \\
\% \\
\end{array}$ & $\begin{array}{c}\mathrm{ER} \\
\% \\
\end{array}$ & $\begin{array}{c}\text { EL } \\
\% \\
\end{array}$ & $\begin{array}{c}\mathrm{ER} \\
\% \\
\end{array}$ & $\begin{array}{l}\mathrm{EL} \\
\% \\
\end{array}$ & $\begin{array}{c}\mathrm{ER} \\
\% \\
\end{array}$ & $\begin{array}{c}\mathrm{EL} \\
\% \\
\end{array}$ & $\begin{array}{c}\mathrm{ER} \\
\% \\
\end{array}$ & $\begin{array}{c}\mathrm{EL} \\
\% \\
\end{array}$ & $\begin{array}{c}\mathrm{ER} \\
\% \\
\end{array}$ \\
\hline $\begin{array}{l}\text { Instrucciones } \\
\text { claras y precisas }\end{array}$ & 37,27 & 27,08 & 41,90 & 33,10 & 16,90 & 30,32 & 3,01 & 7,64 & 0,93 & 1,85 \\
\hline $\begin{array}{l}\text { Seguimiento de } \\
\text { actividades }\end{array}$ & 29,40 & 26,16 & 37,27 & 31,94 & 23,61 & 26,62 & 6,94 & 11,81 & 2,78 & 3,4 \\
\hline $\begin{array}{l}\text { Flexibilidad } \\
\text { académica }\end{array}$ & 25,93 & 29,40 & 29,63 & 31,71 & 27,55 & 22,92 & 11,34 & 10,19 & 5,56 & 5,79 \\
\hline $\begin{array}{l}\text { Diversificación de } \\
\text { canales de } \\
\text { comunicación }\end{array}$ & 24,07 & 28,70 & 25,93 & 28,94 & 32,18 & 28,47 & 12,73 & 10,42 & 5,09 & 3,47 \\
\hline $\begin{array}{l}\text { Inmediatez en las } \\
\text { respuestas del } \\
\text { profesor }\end{array}$ & 27,55 & 23,84 & 37,96 & 30,56 & 25,69 & 28,24 & 7,64 & 14,58 & 1,16 & 2,78 \\
\hline $\begin{array}{l}\text { Clima de } \\
\text { confianza }\end{array}$ & 36,81 & 32,87 & 35,88 & 32,41 & 20,83 & 25,00 & 5,32 & 7,87 & 1,16 & 1,85 \\
\hline
\end{tabular}

Nota. EL = Enseñanza en línea, ER = Enseñanza remota de emergencia

De acuerdo con la Tabla 2, los cuatro aspectos relacionados con la instrucción directa en el escenario EL que los estudiantes consideran más favorables son las instrucciones claras y precisas $(41,90 \%)$ que reciben por parte del profesor, la inmediatez de sus respuestas $(37,96 \%)$, el seguimiento de las actividades $(37,27 \%)$ y el clima de confianza generado por el profesor $(36,81 \%)$, mientras que los aspectos considerados como menos favorables son la diversificación de canales de comunicación $(12,73 \%)$ y la flexibilidad académica $(11,34 \%)$.

Por su parte, en el escenario ER no se observan valores superiores al 35\% en los rangos considerados como más favorables y, en cambio, los cuatro aspectos que los estudiantes consideran como menos favorables con respecto a la instrucción directa son la inmediatez de las respuestas del profesor $(14,58 \%)$, el seguimiento que hace de las actividades $(11,81 \%)$, la diversificación de canales de comunicación $(10,42 \%)$ y la flexibilidad académica $(10,19 \%)$.

En la Figura 2, que conserva el formato de presentación de la figura anterior, se presenta el contraste entre las experiencias de los estudiantes en torno a la instrucción directa en los escenarios EL y ER.

Contraste de experiencias de estudiantes universitarios en dos escenarios educativos: enseñanza en línea vs. enseñanza remota de emergencia. Niño, Castellanos-Ramírez y Patrón. 


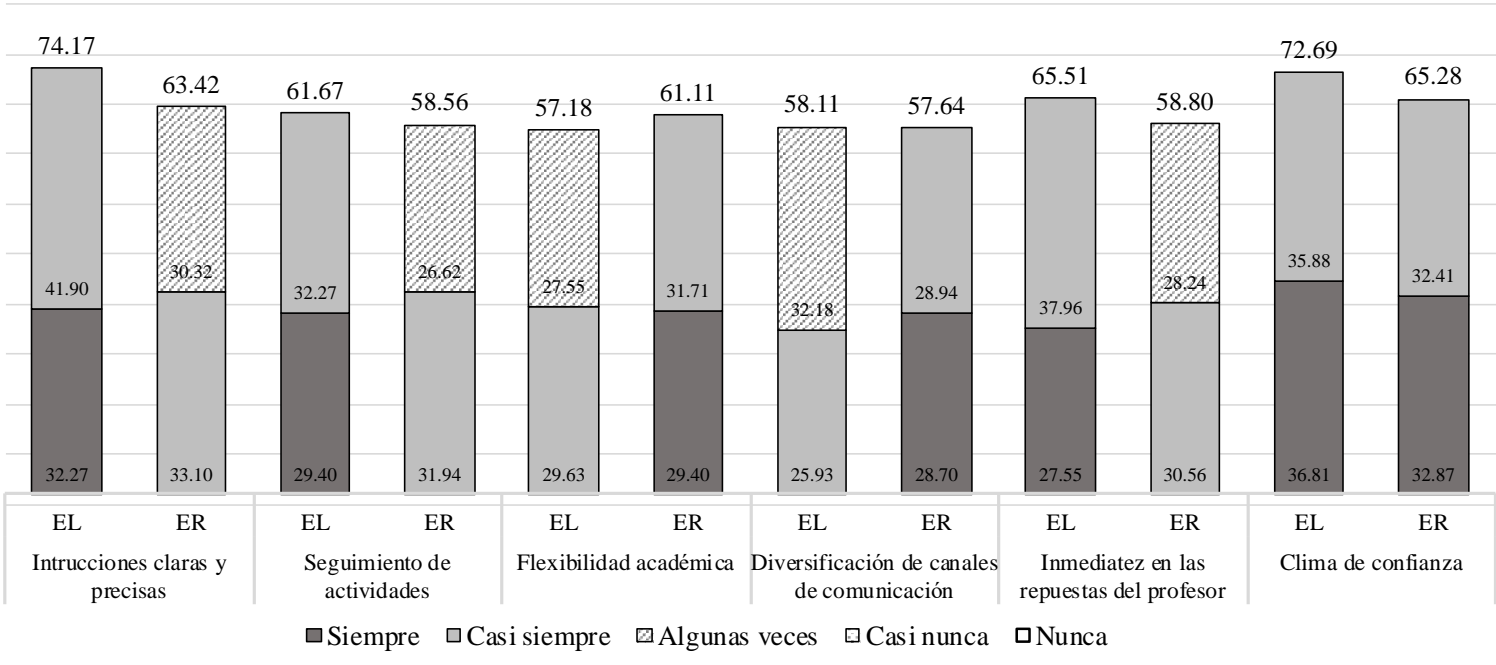

Figura 2. Contraste de las experiencias de los estudiantes en torno a la instrucción directa en los escenarios de enseñanza en línea (EL) y enseñanza remota de emergencia (ER).

Según los datos de la Figura 2, los aspectos de la instrucción directa que los estudiantes consideran más favorables, nuevamente, son los que se llevan a cabo en el escenario EL, sobre todo, las instrucciones claras y precisas que reciben por parte del profesor $(74,17 \%)$ y el clima de confianza que genera $(72,69 \%)$; estos aspectos destacan por presentar porcentajes arriba del 70\% y, además, porque son el resultado de sumar porcentajes de los rangos siempre y casi siempre. En contraste, aunque el escenario ER presenta porcentajes arriba del $60 \%$ en ambos aspectos, solamente el que refiere al clima de confianza $(65,28 \%)$ es el resultado de sumar porcentajes de los rangos siempre y casi siempre.

Sobre el resto de los ítems, los porcentajes totales y el tipo de rangos son más favorables para la inmediatez en las respuestas del profesor $(65,51 \%)$ y el seguimiento de actividades $(61,67 \%)$ del escenario EL y, en cambio, la flexibilidad académica $(61,11 \%)$ y la diversificación que el profesor realiza sobre los canales de comunicación $(57,64 \%)$ son más favorables en el escenario ER; sobre este último aspecto, aunque el porcentaje es ligeramente mayor en el escenario EL $(58,11 \%)$ se concentra en los rangos casi siempre y algunas veces, mientras que el porcentaje del escenario ER $(57,64 \%)$ se centra en los rangos siempre y casi siempre.

Por lo que respecta a la evaluación de los aprendizajes, la Tabla 3 presenta los porcentajes correspondientes a las respuestas de los estudiantes en torno a dicha categoría en los escenarios EL y ER. Cabe recordar que en las columnas de los rangos siempre y casi siempre se distinguen los valores iguales o superiores (celdas con datos en negritas) a un porcentaje equivalente al 35\%; en las columnas de los rangos casi nunca y nunca se distinguen los valores iguales o superiores (celdas con datos en negritas) a un porcentaje equivalente al $10 \%$.

Contraste de experiencias de estudiantes universitarios en dos escenarios educativos: enseñanza en línea vs. enseñanza remota de emergencia. Niño, Castellanos-Ramírez y Patrón. 
RED. Revista de Educación a Distancia. Núm. 65, Vol. 21. Artíc. 7, 08-01-2021

DOI: https://doi.org/10.6018/red.440731

Tabla 3

Experiencias en torno a la evaluación de los aprendizajes

\begin{tabular}{|c|c|c|c|c|c|c|c|c|c|c|}
\hline \multirow{2}{*}{ Ítems } & \multicolumn{2}{|c|}{ Siempre } & \multicolumn{2}{|c|}{ Casi siempre } & \multicolumn{2}{|c|}{$\begin{array}{c}\text { Algunas } \\
\text { veces }\end{array}$} & \multicolumn{2}{|c|}{ Casi nunca } & \multicolumn{2}{|c|}{ Nunca } \\
\hline & $\begin{array}{l}\text { EL } \\
\%\end{array}$ & $\begin{array}{c}\text { ER } \\
\%\end{array}$ & $\begin{array}{l}\text { EL } \\
\%\end{array}$ & $\begin{array}{c}\text { ER } \\
\%\end{array}$ & $\begin{array}{l}\text { EL } \\
\%\end{array}$ & $\begin{array}{c}\text { ER } \\
\%\end{array}$ & $\begin{array}{c}\text { EL } \\
\%\end{array}$ & $\begin{array}{c}\mathrm{ER} \\
\%\end{array}$ & $\begin{array}{l}\text { EL } \\
\%\end{array}$ & $\begin{array}{c}\mathrm{ER} \\
\%\end{array}$ \\
\hline $\begin{array}{l}\text { Claridad en los } \\
\text { criterios generales } \\
\text { para la evaluación }\end{array}$ & 47,26 & 30,35 & 33,33 & 31,59 & 14,43 & 23,63 & 3,98 & 10,20 & 1,00 & 4,23 \\
\hline $\begin{array}{l}\text { Claridad en los } \\
\text { criterios } \\
\text { específicos o } \\
\text { rúbricas de } \\
\text { evaluación }\end{array}$ & 44,28 & 32,59 & 35,32 & 27,61 & 15,67 & 25,12 & 2,74 & 10,70 & 1,99 & 3,98 \\
\hline $\begin{array}{l}\text { Evaluación según } \\
\text { la dificultad de las } \\
\text { tareas y tiempo } \\
\text { invertido en ellas }\end{array}$ & 29,60 & 21,64 & 41,79 & 32,34 & 21,89 & 32,09 & 4,73 & 10,45 & 1,99 & 3,48 \\
\hline $\begin{array}{l}\text { Retroalimentación } \\
\text { sobre las tareas } \\
\text { elaboradas }\end{array}$ & 25,37 & 19,15 & 30,85 & 21,89 & 28,11 & 32,09 & 10,45 & 19,65 & 5,22 & 7,21 \\
\hline $\begin{array}{l}\text { Retroalimentación } \\
\text { en un tiempo } \\
\text { razonable (antes de } \\
\text { dar inicio a la } \\
\text { siguiente } \\
\text { actividad) }\end{array}$ & 26,37 & 18,66 & 31,59 & 23,63 & 25,12 & 27,11 & 11,19 & 21,39 & 5,72 & 9,20 \\
\hline $\begin{array}{l}\text { Retroalimentación } \\
\text { clara y útil para } \\
\text { mejorar la } \\
\text { realización de } \\
\text { trabajos futuros }\end{array}$ & 27,11 & 20,40 & 32,59 & 22,89 & 26,12 & 28,86 & 8,96 & 18,16 & 5,22 & 9,70 \\
\hline
\end{tabular}

Nota. EL = Enseñanza en línea, ER = Enseñanza remota de emergencia

En torno a la evaluación de los aprendizajes en las asignaturas del escenario EL, la Tabla 3 muestra que los tres aspectos considerados por los estudiantes como más favorables son la claridad tanto de los criterios generales $(47,26 \%)$ como de los criterios específicos $(44,28 \%)$ de evaluación y sus resultados según la dificultad de las tareas y el tiempo invertido en ellas (41,79\%); en contraste, los dos aspectos considerados como menos favorables son la retroalimentación que reciben en un tiempo razonable $(11,19 \%)$ y la simple retroalimentación de las tareas $(10,45 \%)$ independientemente del momento en el que se reciban.

En cambio, en el escenario ER, una vez más, no se observan valores superiores al $35 \%$ en los rangos considerados como más favorables (siempre y casi siempre) y, en cambio, todos los aspectos son considerados por los estudiantes como menos favorables (todos

Contraste de experiencias de estudiantes universitarios en dos escenarios educativos: enseñanza en línea vs. enseñanza remota de emergencia. Niño, Castellanos-Ramírez y Patrón. 
presentan porcentajes arriba del 10\%), sobre todo los relacionados con la recepción de retroalimentaciones.

La Figura 3 presenta el contraste entre las experiencias de los estudiantes sobre la evaluación de los aprendizajes en los escenarios EL y ER; vale la pena recordar que en cada barra se muestra la suma de los dos porcentajes más altos obtenidos en cada ítem.

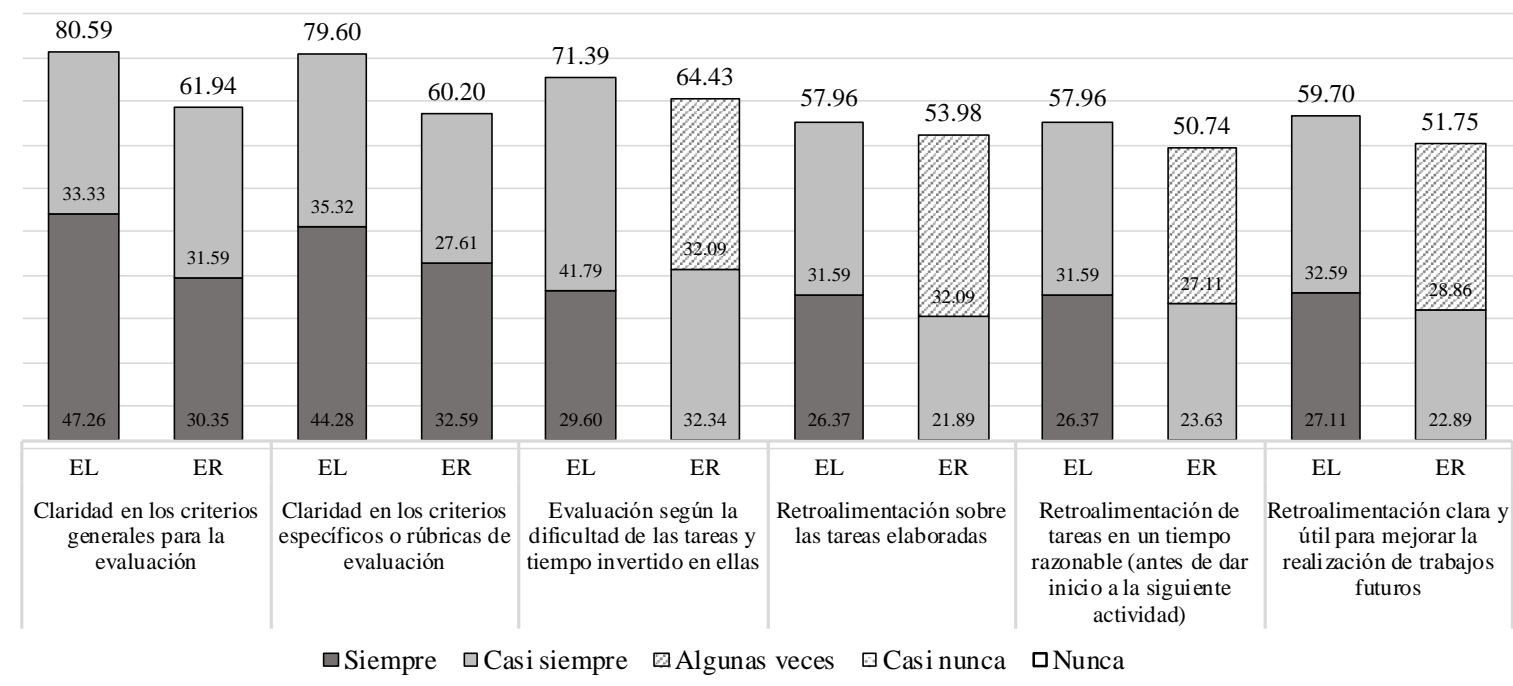

Figura 3. Contraste de las experiencias de los estudiantes sobre la evaluación de los aprendizajes en los escenarios de enseñanza en línea (EL) y enseñanza remota de emergencia (ER).

De acuerdo con la Figura 3, los aspectos de la evaluación de los aprendizajes que los estudiantes consideran más favorables son los que se llevan a cabo en el escenario EL, especialmente, la claridad de los criterios generales $(80,59 \%)$ y específicos $(79,60 \%)$ de evaluación y sus resultados según la dificultad de las tareas y el tiempo invertido en ellas $(71,39 \%)$; pese a que estos tres aspectos también se valoran favorablemente en el escenario ER, los porcentajes de la claridad de los criterios generales $(61,94 \%)$ y específicos (60.20\%) de evaluación están prácticamente $20 \%$ por debajo de lo observado en el escenario EL, mientras que el porcentaje de los resultados de la evaluación $(64,43 \%)$ se centra en los rangos casi siempre y algunas veces.

Aunque en el resto de los ítems no se observan diferencias contundentes entre los porcentajes totales, sí se distinguen diferencias en el tipo de rangos de cada escenario. En detalle, mientras que los tres porcentajes relativos a la recepción de retroalimentaciones en el escenario EL son el resultado de sumar porcentajes de los rangos siempre y casi siempre, en el escenario ER estos porcentajes se centran en los rangos casi siempre y algunas veces.

La Tabla 4 muestra los porcentajes correspondientes a las respuestas de los estudiantes en torno a su experiencia directa sobre la realización de asignaturas en los escenarios EL y ER; en esta se conserva el formato de presentación de la tabla anterior.

Contraste de experiencias de estudiantes universitarios en dos escenarios educativos: enseñanza en línea vs. enseñanza remota de emergencia. Niño, Castellanos-Ramírez y Patrón. 
RED. Revista de Educación a Distancia. Núm. 65, Vol. 21. Artíc. 7, 08-01-2021

DOI: https://doi.org/10.6018/red.440731

Tabla 4

Experiencia global de los estudiantes sobre su participación en las asignaturas de cada escenario

\begin{tabular}{|c|c|c|c|c|c|c|c|c|c|c|}
\hline \multirow{2}{*}{ Ítems } & \multicolumn{2}{|c|}{ Siempre } & \multicolumn{2}{|c|}{ Casi siempre } & \multicolumn{2}{|c|}{$\begin{array}{l}\text { Algunas } \\
\text { veces }\end{array}$} & \multicolumn{2}{|c|}{ Casi nunca } & \multicolumn{2}{|c|}{ Nunca } \\
\hline & $\begin{array}{l}\text { EL } \\
\%\end{array}$ & $\begin{array}{c}\text { ER } \\
\%\end{array}$ & $\begin{array}{l}\text { EL } \\
\%\end{array}$ & $\begin{array}{c}\text { ER } \\
\%\end{array}$ & $\begin{array}{c}\mathrm{EL} \\
\%\end{array}$ & $\begin{array}{c}\mathrm{ER} \\
\%\end{array}$ & $\begin{array}{c}\text { EL } \\
\%\end{array}$ & $\begin{array}{c}\text { ER } \\
\%\end{array}$ & $\begin{array}{c}\text { EL } \\
\%\end{array}$ & $\begin{array}{c}\text { ER } \\
\%\end{array}$ \\
\hline $\begin{array}{l}\text { Diseño de las } \\
\text { actividades }\end{array}$ & 27,64 & 17,09 & 44,47 & 33,17 & 24,62 & 34,17 & 2,51 & 12,06 & 0,75 & 3,52 \\
\hline $\begin{array}{l}\text { Desempeño de los } \\
\text { profesores }\end{array}$ & 32,41 & 17,09 & 42,21 & 34,42 & 21,61 & 39,20 & 3,02 & 7,29 & 0,75 & 2,01 \\
\hline $\begin{array}{l}\text { Carga de trabajo y } \\
\text { nivel de exigencia }\end{array}$ & 23,87 & 11,81 & 45,73 & 27,89 & 25,88 & 34,67 & 3,77 & 18,59 & 0,75 & 7,04 \\
\hline $\begin{array}{l}\text { Evaluación de los } \\
\text { aprendizajes }\end{array}$ & 25,35 & 13,82 & 44,22 & 34,17 & 25,41 & 32,41 & 4,52 & 15,08 & 0,50 & 4,52 \\
\hline Desempeño propio & 37,69 & 24,37 & 42,46 & 39,20 & 17,09 & 24,87 & 1,76 & 8,04 & 1,01 & 3,52 \\
\hline $\begin{array}{l}\text { Estimación global } \\
\text { del escenario }\end{array}$ & 29,40 & 15,08 & 40,20 & 28,89 & 26,63 & 36,93 & 3,02 & 14,07 & 0,75 & 5,03 \\
\hline
\end{tabular}

Nota. EL = Enseñanza en línea, ER = Enseñanza remota de emergencia

De acuerdo con los datos de la Tabla 4, los estudiantes consideran favorables los seis aspectos relativos a su experiencia global al participar en las asignaturas del escenario EL, ya que los porcentajes superan, incluso, el 40\%; de manera opuesta, en las columnas de los rangos menos favorables (casi nunca y nunca) no se observan porcentajes iguales o superiores al $10 \%$.

A diferencia de los tres aspectos anteriores del escenario ER en los que no aparecieron valores superiores el 35\% en los rangos siempre y casi siempre, esta vez aparece un aspecto considerado por los estudiantes como más favorable, que es el desempeño propio $(39,20 \%)$; por el contrario, los cuatro aspectos considerados como menos favorables son la carga de trabajo y nivel de exigencia $(18,59 \%)$, la evaluación de los aprendizajes $(15,08 \%)$, la estimación global de este escenario $(14,07 \%)$ y el diseño de las actividades $(12,06 \%)$.

En la Figura 4, que conserva el formato de presentación de la figura anterior, se presenta el contraste entre la experiencias global de los estudiantes sobre su participación en las asignaturas de los escenarios EL y ER.

Contraste de experiencias de estudiantes universitarios en dos escenarios educativos: enseñanza en línea vs. enseñanza remota de emergencia. Niño, Castellanos-Ramírez y Patrón. 


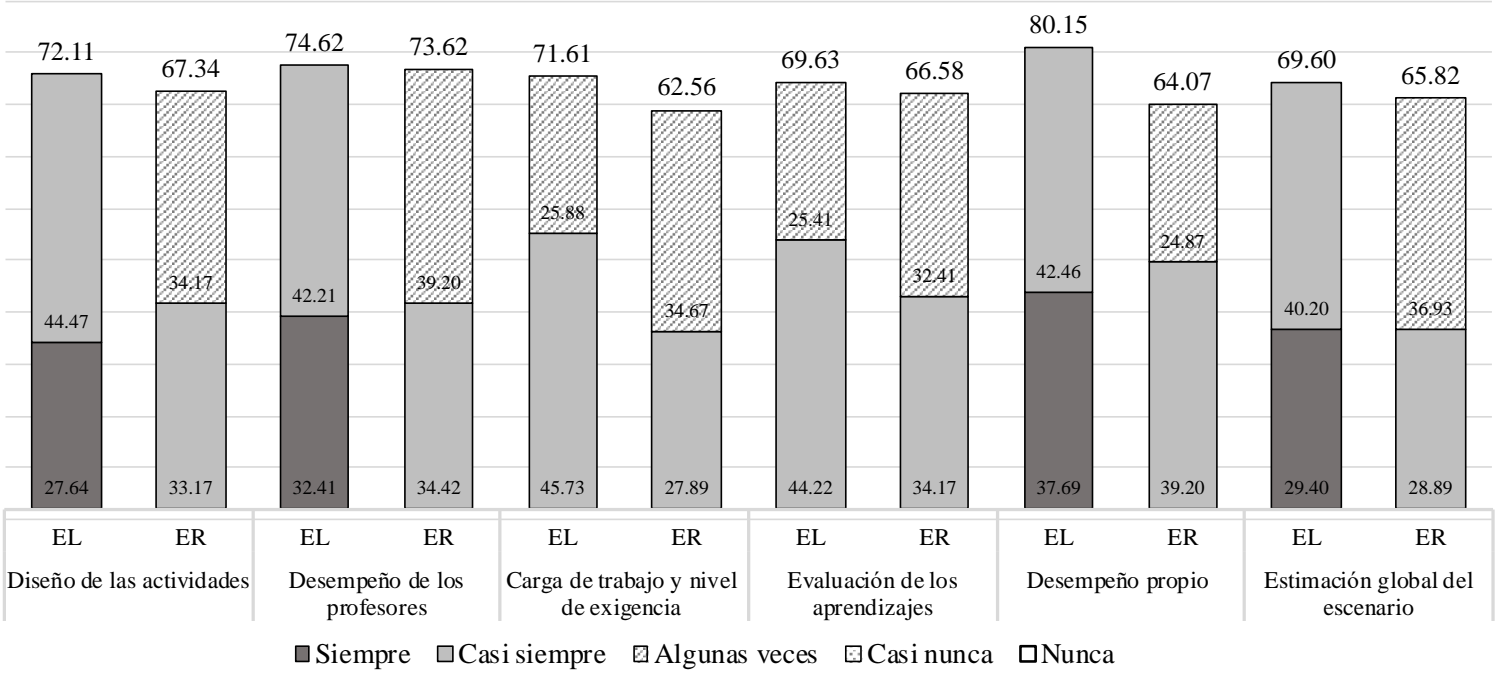

Figura 4. Contraste de las experiencias globales de los estudiantes sobre su participación en las asignaturas de los escenarios de enseñanza en línea (EL) y enseñanza remota de emergencia (ER).

Como se observa en la Figura 4, los aspectos relacionados con las experiencias globales de los estudiantes al participar en las asignaturas que se consideran más favorables son los que se desarrollan en el escenario EL. En detalle, los aspectos que más destacan son la valoración del desempeño propio (80,15\%), el desempeño de los profesores $(74,62 \%)$, el diseño de las actividades $(72,11 \%)$ y la estimación global del escenario EL $(69,60 \%)$; cabe destacar que estos porcentajes, arriba incluso del $70 \%$, son el resultado de sumar porcentajes de los rangos casi siempre y siempre; por el contrario, pese a que en el escenario ER estos aspectos presentan porcentajes arriba del $60 \%$, excepto en la valoración del desempeño de los profesores $(71,61 \%)$, son el resultado de sumar porcentajes de los rangos casi siempre y algunas veces.

En relación a los dos aspectos restantes, aunque no se observan diferencias entre el tipo de rangos de cada escenario, sí hay diferencias en los porcentajes de cada rango; en detalle, la carga de trabajo y nivel de exigencia es más o menos favorable para el 71,61\% de los estudiantes en el escenario EL y para el 62,56\% en el escenario ER, sin embargo, el escenario EL destaca porque el $45,73 \%$ corresponde al rango casi siempre, mientras que en el escenario ER el porcentaje más alto $(34,67 \%)$ se concentra en el rango algunas veces. Algo similar se observa con respecto a la evaluación de los aprendizajes, ya que este aspecto es más o menos favorable para el 69,63\% de los estudiantes en el escenario EL y para el $66,58 \%$ en el escenario ER, pero el escenario EL destaca porque el porcentaje más alto $(44,22 \%)$ está $10 \%$ arriba del porcentaje más alto $(34,17 \%)$ del escenario ER.

Finalmente, de acuerdo con la prueba t de Student para muestras relacionadas en la que se compararon los reportes de los participantes para cada categoría (instrucción, diseño, evaluación, valoración) en los escenarios enseñanza en línea y enseñanza remota de emergencia, los resultados del análisis estadístico inferencial realizado son los siguientes.

Contraste de experiencias de estudiantes universitarios en dos escenarios educativos: enseñanza en línea vs. enseñanza remota de emergencia. Niño, Castellanos-Ramírez y Patrón. 
Para la categoría de diseño de actividades en la modalidad de enseñanza en línea $(\mathrm{M}=$ 22.242; $\mathrm{DE}=3.793)$ y remota de emergencia $(\mathrm{M}=20.398 ; \mathrm{DE}=4.418)$ se encontraron diferencias significativas entre los reportes de los participantes $(t(300)=8.397, \mathrm{p}=.001$, $d=0.445, \beta-1=1$, IC $[1.411,2.275])$. Para la categoría de instrucción directa en el escenario en línea $(M=23.086 ; \mathrm{DE}=4.817)$ y en emergencia $(\mathrm{M}=22.711 ; \mathrm{DE}=5.376)$, no se encontraron diferencias significativas entre los reportes de los participantes $(t(300)$ $=1.452, \mathrm{p}=.147, d=0.073, \beta-1=.244$, IC $[-0.1333,0.884])$. En tanto que, para la categoría evaluación en el escenario en línea $(\mathrm{M}=23.212 ; \mathrm{DE}=4.971)$ y en emergencia $(\mathrm{M}=20.883 ; \mathrm{DE}=5.826)$ se encontraron diferencias significativas entre los reportes $(t(300)=0.098, \mathrm{p}=.001, d=0.427, \beta-1=1, \mathrm{IC}[1.762,2.894])$.

Ahora bien, al comparar los reportes de los participantes para la categoría de valoración de experiencias en el escenario en línea $(\mathrm{M}=23.867 ; \mathrm{DE}=4.273)$ y en emergencia $(\mathrm{M}=$ 20.760; $\mathrm{DE}=5.241)$, se encontró que existen diferencias significativas entre los reportes de las modalidades en línea y en emergencia $(t(300)=10.801, \mathrm{p}=.001, d=0.643, \beta-1=$ $1, \mathrm{IC}[2.540,3.672])$.

\section{DISCUSIÓN Y CONCLUSIONES}

Aun cuando se ha constatado que el cierre de escuelas ayuda a controlar la transmisión comunitaria ante los brotes de una epidemia (Brown et al., 2011), la emergencia sanitaria por la Covid-19 ha impuesto múltiples retos y desafíos para las instituciones educativas que, en el intento por dar continuidad académica a los programas educativos, han migrado abruptamente de una modalidad presencial a una modalidad en línea (Sánchez et al., 2020). Hasta el momento, de acuerdo con los trabajos revisados, las investigaciones sobre enseñanza remota de emergencia se han centrado en analizar los desafíos que los sistemas educativos, docentes o estudiantes han presentado ante la emergencia por la Covid-19.

Sin embargo, mientras que unos se centran en el papel de la tecnología para transitar de una modalidad educativa a otra valorando el nivel de uso o dominio que tienen los docentes o los estudiantes sobre los recursos tecnológicos (Basilaia y Kvavadze, 2020; Gazca, 2020; Grammegna, 2020; Subedi et al., 2020), otros van más allá de esta dimensión meramente tecnológica y se centran en mirar cómo es que determinadas prácticas se ajustaron a este nuevo escenario educativo (Amaya et al., 2021; De la Calle et al, 2021; Gomes et al., 2021; García-de-Paz y Santana, 2021; Rajab et al., 2020). Bajo este marco, el trabajo ha estado presidido por el objetivo de explorar las experiencias de una población de estudiantes universitarios en torno a la instrucción recibida por parte de sus profesores en un escenario de enseñanza en línea, antes de la Covid-19, y en un escenario de enseñanza remota de emergencia, durante la Covid-19.

En primer lugar, grosso modo, los datos porcentuales muestran que, efectivamente, las experiencias de los estudiantes son más favorables en el escenario de enseñanza en línea y menos favorables en el escenario de enseñanza remota de emergencia. En concreto, estas experiencias favorables están relacionadas con el diseño de las actividades, la instrucción directa del profesor, la evaluación de los aprendizajes y la propia participación de los estudiantes en las asignaturas del escenario de enseñanza en línea; por el contrario, en el escenario de enseñanza remota de emergencia, las cuatro categorías (instrucción,

Contraste de experiencias de estudiantes universitarios en dos escenarios educativos: enseñanza en línea vs. enseñanza remota de emergencia. Niño, Castellanos-Ramírez y Patrón. 
diseño, evaluación y participación en asignaturas) sobre las que los estudiantes valoraron sus experiencias, habitualmente, siempre muestran porcentajes más bajos.

Aunado a lo anterior, se constató que las medias para los reportes fueron mayores para el escenario de enseñanza en línea en comparación con el escenario de enseñanza remota de emergencia para las cuatro categorías, aunque solo se hayan encontrado diferencias estadísticamente significativas al comparar tres de estas, lo cual implica que los participantes experimentaron el proceso formativo de forma más positiva en el escenario de enseñanza en línea.

Los resultados anteriores apoyan, hasta cierto punto, los señalamientos de diversos autores sobre el potencial de la educación en línea, es decir, que este tipo de escenarios son especialmente favorables para el aprendizaje de los estudiantes cuando existen diseños instruccionales pautados (Rozitis, 2017; Park y Lou, 2017), con un alto nivel de especialización de los profesores a nivel tecnopedagógico (Comas et al., 2012, Watson et al., 2017) y cuando se ponen en marcha procesos de evaluación continua que fomentan el aprendizaje individual y colaborativo (Alvarado, 2014; Amaya et al., 2021; Shintani, 2016).

En segundo lugar, con respecto a la categoría relacionada con el diseño de las actividades, los resultados evidencian que, dentro del escenario de enseñanza remota de emergencia, las experiencias valoradas por los estudiantes como menos favorables se dan en torno al volumen de tareas, las estrategias o recursos didácticos empleados como apoyo al aprendizaje, la relevancia de los contenidos y la propia organización de las actividades. Estos resultados podrían estar asociados con las adecuaciones tal vez improvisadas, abruptas o contingentes hechas sobre diseños instruccionales inicialmente pensados para una modalidad presencial, que al migrar a una modalidad en línea perdieron la estrecha relación entre sus elementos. De hecho, el estudio de Gomes et al. (2021) evidencia que no solo el diseño instruccional de los cursos debe alinearse a las características de este nuevo escenario educativo, sino que los materiales didácticos también deben ajustarse, ya sea en formato de Microcontenido (MC), que resulta adecuado para contenidos de baja densidad, o en formato LongForm, que es más apropiado para contenido complejo trabajado típicamente en los cursos en línea.

Además, de acuerdo con Rodríguez y Gallardo (2019), los buenos diseños instruccionales en la educación en línea se caracterizan por desarrollar los procesos de diseño instruccional a priori, de tal manera que el estudiante conozca oportunamente lo que va a aprender y el cómo demostrará su desempeño; la premisa anterior confirma, hasta cierto punto, que el diseño de las actividades en el escenario de enseñanza en línea sea valorado más favorable por los participantes de este estudio, sobre todo la organización de las actividades, la asignación de tiempo para realizarlas, la complejidad progresiva de las tareas y la diversidad de estrategias y recursos didácticos.

En tercer lugar, en torno a la instrucción directa de los profesores y su papel en el escenario de enseñanza remota de emergencia, se encontró que los aspectos menos favorables son la inmediatez de sus respuestas para resolver las dudas de los estudiantes y el seguimiento que realizan de las actividades. Lo anterior podría deberse a la carga de trabajo y las dificultades tecnológicas que los profesores experimentaron al incursionar precipitadamente a un nuevo escenario de enseñanza, tal como señalan Bozkurt y Sharma

Contraste de experiencias de estudiantes universitarios en dos escenarios educativos: enseñanza en línea vs. enseñanza remota de emergencia. Niño, Castellanos-Ramírez y Patrón. 
(2020), Gleen (2020) y Muller y Goldenber (2020), quienes han evidenciado que durante la emergencia sanitaria, una de las grandes limitaciones de los profesores ha sido, justamente, la falta de experiencia y su escasa formación en el uso de plataformas tecnológicas y la facilitación del aprendizaje en línea.

De manera complementaria, los hallazgos de Amaya et al. (2021) señalan la importancia de que, además de capacitar a los profesores en el manejo de determinadas plataformas tecnológicas, también se debe reparar en el desarrollo de las habilidades y competencias didácticas virtuales que integren las potencialidades de la tecnología con los contenidos y los conocimientos pedagógicos.

En cambio, del escenario de enseñanza en línea, los aspectos que los estudiantes consideran más favorables son las instrucciones claras y precisas que reciben por parte de los profesores, la inmediatez de sus respuestas, el seguimiento que hacen de las actividades y el clima de confianza que generan. Estos resultados confirman los hallazgos de Watson et al. (2017), puesto que las estrategias instruccionales principales que ayudan a los estudiantes en sus procesos de aprendizaje se relacionan con alguna forma de interacción con sus profesores, por ejemplo, estar disponible y receptivo a los estudiantes, interactuar y participar con ellos o fomentar la interacción y comunicación. De hecho, en el trabajo de Rajab et al. (2020), la comunicación entre profesores y estudiantes también aparece como un elemento imprescindible por mejorarse de cara al desarrollo de los cursos académicos durante la pandemia.

En cuarto lugar, en coincidencia con Dorrego (2016), el diseño de la evaluación debe ocupar un papel primordial en cualquier modalidad instruccional para guardar estrecha relación con los procesos de enseñanza y de aprendizaje; no obstante, los resultados evidencian que en el caso del escenario de enseñanza remota de emergencia no se le prestó la atención debida, ya que los estudiantes valoraron menos favorables aspectos como la claridad en los criterios generales y específicos de evaluación, los resultados de la evaluación según la dificultad de las tareas y el tiempo invertido, las retroalimentaciones hechas a las tareas, el tiempo razonable en el que se reciben y su utilidad para mejorar trabajos posteriores. Este señalamiento también aparece en los trabajos de García-de-Paz y Santana (2021) y Rajab et al. (2020), quienes coinciden en la urgencia de mejorar la función evaluativa en este nuevo escenario, ya sea mediante retroalimentaciones claras y precisas que reorienten la actividad de los estudiantes o priorizando la evaluación de los procesos sobre la evaluación de los resultados.

Cabe destacar que los aspectos relacionados con la recepción de retroalimentaciones también aparecieron, en menor medida, como menos favorables en el escenario de enseñanza en línea, por lo que cabe plantearse si las prácticas evaluativas que se han desarrollado en este escenario consideran la parte postevaluativa de comunicación de los resultados a los estudiantes (Barberá, 2016) y, de existir, si cumplen solo una función normativa de evaluación o desarrollan también una función psicopedagógica.

En quinto lugar, otra conclusión interesante se deriva de la única valoración favorable que los estudiantes realizan sobre sus experiencias al participar en las asignaturas del escenario de enseñanza remota de emergencia; en efecto, dentro de todos los aspectos, los estudiantes valoran que su propio desempeño en las asignaturas -participación, asistencia, puntualidad y cumplimiento de las tareas- es el más favorable, en detrimento

Contraste de experiencias de estudiantes universitarios en dos escenarios educativos: enseñanza en línea vs. enseñanza remota de emergencia. Niño, Castellanos-Ramírez y Patrón. 
del diseño instruccional, el desempeño de los profesores, la carga de trabajo y nivel de exigencia, así como de la evaluación de los aprendizajes.

Bajo la consideración de que los procesos de aprendizaje en línea son propicios para desarrollar habilidades de autorregulación (Niño et al., 2019), se puede concluir que, ante la ausencia de un diseño instruccional ad hoc al escenario de enseñanza remota de emergencia y, por ende, de la intervención contingente del profesor, los estudiantes desarrollaron -forzosamente- procesos de autorregulación o aprendizaje autónomo (Moreno y Cárdenas, 2012) para concluir satisfactoriamente el ciclo escolar, aun cuando lo deseable es que dichos procesos sean promovidos desde el diseño instruccional y gracias a la mediación del profesor. Sin duda, este es un aspecto en el que, a corto plazo, debe profundizarse con el fin de rescatar las estrategias puestas en marcha por los estudiantes, ya que son elementos que deben ser recuperados para la mejora de los diseños instruccionales.

Aunque los resultados permiten confirmar sendas diferencias sobre las experiencias de los estudiantes entre los escenarios de enseñanza en línea y de enseñanza remota de emergencia, ponen también de manifiesto un par de limitaciones y carencias que son, a la vez, vías de acción para futuros trabajos.

A nivel metodológico, a partir de los contrastes entre las condiciones en línea y en emergencia por medio de la prueba $t$ de Student para muestras relacionadas, se encontraron tamaños del efecto medianos y potencias estadísticas de uno para las categorías de diseño, evaluación y participación. Esto brinda bases para proponer que las diferencias entre los reportes de lo que experimentaron los participantes en los dos escenarios educativos podrían replicarse con los estudiantes de otros programas educativos ofertados por la Facultad de Ciencias Humanas.

De manera complementaria, la utilización de métodos cualitativos -como la triangulación y el contraste con otros informantes- puede contribuir a la profundización de las experiencias, tanto de estudiantes como de profesores, desde un sentir más individual y contextualizado de cada escenario reconociendo, de esta manera, las distintas realidades educativas frente a la pandemia y, sobre todo, los medios y estrategias empleadas para concluir de la manera más satisfactoria posible el ciclo escolar.

Finalmente, aunque los resultados dan cuenta de los aspectos concretos que los estudiantes valoran más o menos favorables en cada uno de los dos escenarios, también interpelan un conjunto de preguntas que, por la naturaleza de este estudio, no es posible responder; por ejemplo, en el escenario de enseñanza en línea cabe preguntarse por los factores asociados al sentido o la relevancia que los estudiantes confieren -o no- a los contenidos que abordan en las distintas asignaturas o bien por el impacto de las prácticas de evaluación y retroalimentación sobre los procesos de aprendizaje. De manera similar, en el escenario de enseñanza remota de emergencia valdría la pena evidenciar las buenas prácticas de los profesores en ese tránsito de la modalidad presencial a la modalidad en línea y su relación con los procesos de aprendizaje o, por ejemplo, conocer los desafíos que enfrentaron los profesores -en distintos niveles- para adaptarse a esta modalidad y si hubo un acompañamiento de las instituciones a las que están adscritos para realizar tales adaptaciones.

Contraste de experiencias de estudiantes universitarios en dos escenarios educativos: enseñanza en línea vs. enseñanza remota de emergencia. Niño, Castellanos-Ramírez y Patrón. 
Sin duda, la pandemia por la Covid-19 plantea múltiples retos y múltiples frentes que deben atenderse con prontitud para garantizar, por un lado, la continuidad académica y, por otro lado, la atención de las situaciones de vulnerabilidad vividas por profesores y estudiantes.

Presentación del artículo: 28 de agosto de 2020

Fecha de aprobación: 14 de diciembre de 2020

Fecha de publicación: 08 de enero de 2021

Niño, S., Castellanos-Ramírez, J. C., y Patrón, F. (2021). Contraste de experiencias de estudiantes universitarios en dos escenarios educativos: enseñanza en línea vs. enseñanza remota de emergencia. Revista Educación a Distancia (RED), 21(65). https://doi.org/10.6018/red.440731

\section{Financiación}

Este trabajo no ha recibido ninguna subvención específica de los organismos de financiación en los sectores públicos, comerciales o sin fines de lucro.

\section{REFERENCIAS}

Ahmed, F., Zviedrite, N., y Uzicanin, A. (2018). Effectiveness of workplace social distancing measures in reducing influenza transmission: a systematic review. BMC Public Health, 18, 518. https://doi.org/10.1186/s12889-018-5446-1

Alvarado, M. A. (2014). Retroalimentación en educación en línea: una estrategia para la construcción del conocimiento. Revista Iberoamericana de Educación a Distancia, 17(2), 59-73.https://doi.org/10.5944/ried.17.2.12678

Álvarez, G. (2020, 16 de abril). Covid-19. Cambiar de paradigma educativo. Consejo Mexicano de Investigación Educativa (COMIE). http://www.comie.org.mx/v5/sitio/2020/04/16/covid-19-cambiar-de-paradigmaeducativo/

Amaya, A., Cantú, D., y Marreros, J. G. (2021). Análisis de las competencias didácticas virtuales en la impartición de clases universitarias en línea, durante contingencia del COVID-19. RED. Revista Educación a Distancia, 21(64). http://doi.org/10.6018/red.426371

Barberà, E. (2016). Aportaciones de la tecnología a la e-Evaluación. Revista de Educación a Distancia (RED), 50, 4. http://www.um.es/ead/red/50

Basilaia, G., y Kvavadze, D. (2020). Transition to Online Education in Schools during a SARS-CoV-2 Coronavirus (COVID-19) Pandemic in Georgia. Pedagogical Research, 5(4), 1-9. https://doi.org/10.29333/pr/7937

Contraste de experiencias de estudiantes universitarios en dos escenarios educativos: enseñanza en línea vs. enseñanza remota de emergencia. Niño, Castellanos-Ramírez y Patrón. 
Bloom D., y Cadarette, D. (2019). Infectious disease threats in the twenty-first century: strengthening the global response. Frontiers in immunology, 10, 549. https://doi.org/10.3389/fimmu.2019.00549

Bolliger, D., y Wasilik, O. (2009). Factors influencing faculty satisfaction with online teaching and learning in higher education. Distance Education, 30(1), 103-116. http://doi.org/10.1080/01587910902845949

Booth, P., Guinmard, I., y Lloyd, E. (2017). The perceptions of a situated learning experience mediated by novice teachers' autonomy. The EUROCALL Review, 25(1), 76-91. https://doi.org/10.4995/eurocall.2017.7081

Bozkurt, A., y Sharma, R. (2020). Emergency remote teaching in a time of global crisis due to CoronaVirus pandemic. Asian Journal of Distance Education, 15(1), i-vi. https://doi.org/10.5281/zenodo.3778083

Brown, S., Tai., Bailey, R., Cooley, P., Wheaton, W., Potter, M., Voorhees, R., LeJeune, M., Grefenstette, J., Burke, D., McGlone S., y Lee, B. (2011). Would school closure for the $2009 \mathrm{H} 1 \mathrm{~N} 1$ influenza epidemic have been worth the cost?: a computational simulation of Pennsylvania. BMC Public Health, 11(1), 353. https://doi.org/10.1186/1471-2458-11-353

Buerger, C., y Harris, D. (2015). How Can Decentralized Systems Solve SystemLevel Problems? An Analysis of Market-Driven New Orleans School Reforms. American Behavioral Scientist, $\quad$ 59(10), 1246-1262. https://doi.org/10.1177/0002764215591182

Carlo, J. T., y Chung, W. (2009). Review of schoolclosure as a pandemic mitigation strategy. Texas medicine, 105(7), 21-26. https://www.texmed.org/template.aspx?id=7808

Cervantes, E., y Gutierrez, P. (2020). Resistir la Covid-19. Intersecciones en la Educación de Ciudad Juárez, México. Revista Internacional de Educación para la Justicia Social, 9(3), 7-23. https://doi.org/10.15366/riejs2020.9.3.001

Chan, K. (2005). Control of severe acute respiratory syndrome in Singapore. Environmental health and preventive medicine, 10(5), 255-259. https://doi.org/10.1007/BF02897699

Comas, A., De los Arcos, B., y Mardomingo, R. (2012). Virtual Learning Environments (VLEs) for distance language learning: shifting tutor roles in a contested space for interaction. Computer Assisted Language Learning, 25(2), 129-143. https://doi.org/10.1080/09588221.2011.636055

Compton, L. (2009). Preparing language teachers to teach language online: a look at skills, roles, and responsibilities. Computer Assisted Language Learning, 22(1), 73-99. https://doi.org/10.1080/09588220802613831

Contraste de experiencias de estudiantes universitarios en dos escenarios educativos: enseñanza en línea vs. enseñanza remota de emergencia. Niño, Castellanos-Ramírez y Patrón. 
Daniel, S. (2020). Education and the COVID-19 pandemic. Prospects. https://doi.org/10.1007/s11125-020-09464-3

De la Calle, C., Miró, S., de Dios, T., y de la Rosa, D. (2021). Adaptación de una materia universitaria al aprendizaje en línea en tiempos de COVID-19: una oportunidad de mejora. RED. Revista Educación a Distancia, 21(65). http://doi.org/10.6018/red.449841

Dorrego, E. (2016). Educación a distancia y evaluación del aprendizaje. Revista de

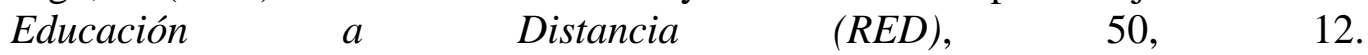
https://revistas.um.es/red/article/view/271241

Esnard, A., Lai, B. S., Wyczalkowski, C., Malmin, N., y Shah, H. (2018). School vulnerability to disaster: examination of school closure, demographic, and exposure factors in Hurricane Ike's winds wath. Natural Hazards, 90(2), 513-535. https://doi.org/10.1007/s11069-017-3057-2

Fernández, M., Herrera, L., Hernández, D., Nolasco, R. y de la Rosa, R. (2020, 1 de abril). Lecciones del Covid-19 para el sistema educativo mexicano. Distancia por tiempos. Blog de educación. https://educacion.nexos.com.mx/?p=2228

Fox, R. (2004). SARS epidemic: Teachers' experiences using ICTs. En R. Atkinson, C. McBeath, D. Jonas-Dwyer y R. Phillips (Eds.), Beyond the confort zone: Proceedings of the 21st ASCILITE Conference (pp. 319-327). ASCILITE. http://www.ascilite.org.au/conferences/perth04/procs/fox.html

García, F., Corell, A., Abella, V., y Grande, M. (2020). La evaluación online en la educación superior en tiempos de la COVID-19. Education in the Knowledge Society, 21, 12. http://doi.org/10.14201/eks.23013

García-de-Paz, S. y Santana, P. J. (2021). La transición a entornos de educación virtual en un contextode emergencia sanitaria: estudio de caso de un equipo docente en Formación Profesional Básica. RED. Revista Educación a Distancia, 21(65). http://doi.org/10.6018/red.450791

Gazca, L. A. (2020). Implicaciones del coronavirus covid-19 en los procesos de enseñanza en la educación superior. RIDE Revista Iberoamericana Para La Investigación $\quad Y \quad$ El Desarrollo Educativo, 11(21). https://doi.org/10.23913/ride.v11i21.753

Glenn (2020, 7 de mayo). From Emergency Remote Teaching to Rigorous Online Learning. $\quad$ EdTech: Focus on Higher Education. https://edtechmagazine.com/higher/article/2020/05/emergency-remote-teachingrigorous-online-learning-perfcon

Gomes, N. S., Martins, R. X., y Azevedo. D. S. (2021). LongForm or Microcontent? An analysis of supports for digital content courseware. RED. Revista Educación a Distancia, 21(65). http://doi.org/10.6018/red.422371

Contraste de experiencias de estudiantes universitarios en dos escenarios educativos: enseñanza en línea vs. enseñanza remota de emergencia. Niño, Castellanos-Ramírez y Patrón. 
Grammegna, A. (2020). The New Challenge of Education Value and Limits of Distance Learning Today. Journal of Education and Culture Studies, 4(2), 59-66. https://doi.org/10.22158/jecs.v4n2p59

Halder, N., Kelso, J. K., y Milne, G. J. (2010). Developing guidelines for school closure interventions to be used during a future influenza pandemic. BMC Infectious Diseases, 10, 221. https://doi.org/10.1186/1471-2334-10-221

Hernández, R., Fernández, C., y Baptista, M. (2014). Metodología de la investigación (6 ${ }^{\mathrm{a}}$ ed.). México: McGraw-Hill.

Hodges, C., Moore, S., Locke, B., Trust, T., y Bond, A. (2020, 27 de marzo). The Difference Between Emergency Remote Teaching and Online Learning. EDUCAUSE. https://er.educause.edu/articles/2020/3/the-difference-betweenemergency-remote-teaching-and-online-learning

Houston, M. (2017). The Experiences of Faculty and Staff at Academic Institutions Preparing Themselves for Academic Continuity after a Disaster: A Phenomenological Study. International Journal of Business and Social Science, 8(7), 7-18. http://ijbssnet.com/journals/Vol_8_No_7_July_2017/2.pdf

Huremovic, D. (2019). Social Distancing, Quarantine, and Isolation. En D. Huremovic (ed.), Psychiatry of Pandemics (pp. 85-94). Springer. https://doi.org/10.1007/978-3-030-15346-5_8

Innes, R. (2020, 7 de abril). The Corona Virus and 'Emergency Remote Teaching' The Data. Bluegrass Institute for Public Policy Solutions (BIPPS). http://www.bipps.org/the-corona-virus-and-emergency-remote-teaching-thedatal

Meyer, K. A., y Wilson, J. L. (2011). The Role of Online Learning in the Emergency Plans of Flagship Institutions. Online Journal of Distance Learning Administration, 14(1). https://www.learntechlib.org/p/52628/

Moreno, O., y Cárdenas, M. G. (2012). Educación a distancia: nueva modalidad, nuevos alumnos. Perfiles de alumnos de Psicología en México. Perfiles educativos, $34(136)$, 118-136. https://doi.org/10.22201/iisue.24486167e.2012.136.31767

Muller, L., y Goldenber, G. (2020). Education in times of crisis: The potential implications of school closures for teachers and students. Chartered College of Teaching. content/uploads/2020/05/CCTReport150520_FINAL.pdf

Murphy, M. (2020). COVID-19 and emergency eLearning: Consequences of the securitization of higher education for post-pandemic pedagogy. Contemporary Security Policy, 41(3), 492-505. https://doi.org/10.1080/13523260.2020.1761749

Contraste de experiencias de estudiantes universitarios en dos escenarios educativos: enseñanza en línea vs. enseñanza remota de emergencia. Niño, Castellanos-Ramírez y Patrón. 
Mutch, C. (2017). Winners and losers: School closures in post-earthquake Canterbury and the dissolution of community. Waikato Journal of Education, 22(1), 73-95. https://doi.org/10.15663/wje.v22i1.543

Niño, S., Castellanos-Ramírez, J. C., y Viloria, E. (2019). Construcción del conocimiento y regulación del aprendizaje en tareas colaborativas asíncronas. Apertura: Revista de Innovación Educativa, 11(1), 6-23. https://doi.org/10.32870/Ap.v11n1.1465

Niño, S., Patrón, F., y Castellanos-Ramírez, J. C. (2020). Diseño y validación de un cuestionario sobre la instrucción del profesor en distintos escenarios de enseñanza. Manuscrito enviado para publicación.

Organización de las Naciones Unidas para la Educación, la Ciencia y la Cultura [UNESCO]. (2020a). Impacto de COVID-19 en la educación. UNESCO. https://es.unesco.org/covid19/educationresponse

Organización de las Naciones Unidas para la Educación, la Ciencia y la Cultura [UNESCO]. (2020b, 24 de abril). Surgen alarmantes brechas digitales en el aprendizaje a distancia. UNESCO. https://es.unesco.org/news/surgenalarmantes-brechas-digitales-aprendizaje-distancia

Pang, X., Zhu, Z., Xu, F., Guo, J., Gong, X., Liu, D., Liu, Z., Chin, D. P., y Feikin, D. R. (2003). Evaluation of control measures implemented in the severe acute respiratory syndrome outbreak in Beijing, 2003. Journal of the American Medical Association, 290(24), 3215-3221. https://doi.org/10.1001/jama.290.24.3215

Park, J., y Luo, H. (2017). Refining a Competency Model for Instructional Designers in the Context of Online Higher Education. International Education Studies, 10(9), 87-98. https://doi.org/10.5539/ies.v10n9p87

Quinn, S. C., y Kumar, S. (2014). Health in equalities and infectious disease epidemics: a challenge for global health security. Biosecurity and Bioterrorism: Biodefense Strategy, Practice, and Science, 12(5), 263-273. https://doi.org/10.1089/bsp.2014.0032

Rajab, M. H., Gazal, A. M., y Alkattan, K. (2020). Challenges to online medical education during the COVID-19 pandemic. Cureus, 12(7). https://doi.org/10.7759/cureus.8966

Rhea, R. (2003). Online Learning Fills Void in Nations Coping With SARS. Education Week, 22(37). https://www.edweek.org/ew/articles/2003/05/21/37sars.h22.html

Rodriguez, V. R., y Gallardo, K. E. (2019). Decisiones en evaluación: ambientes virtuales de posgrado, un estudio ex post-facto. Revista de Educación a Distancia (RED), 19(59). https://doi.org/10.6018/red/59/06

Contraste de experiencias de estudiantes universitarios en dos escenarios educativos: enseñanza en línea vs. enseñanza remota de emergencia. Niño, Castellanos-Ramírez y Patrón. 
Rozitis, C. (2017). Instructional Design Competencies for Online High School Teachers Modifying their own Courses. TechTrends, 61(5), 428-437. https://doi.org/10.1007/s11528-017-0204-2

Sánchez, M., Martínez, A., Torres, R., Servín, M., Hernández, A., Benavides, M., Rendón,V., y Jaimes, C. (2020). Retos educativos durante la pandemia de COVID-19: una encuesta a profesores de la UNAM. Revista Digital Universitaria, 21(3), 1-24. https://www.revista.unam.mx/wpcontent/uploads/a12.pdf

Satpathy, B. y Ali, E. (2020). A study on psychological well-being of final year management students during COVID-19 pandemic lockdown in India. International Journal of Indian Psychology, 8(2), 1-25. https://ijip.in/pdfviewer/?id=21210

Shintani, N. (2016). The effects of computer-mediated synchronous and asynchronous direct corrective feedback on writing: a case study. Computer Assisted Language Learning, 29(3), 517-538. https://doi.org/10.1080/09588221.2014.993400

Subedi, S., Nayaju, S., Subedi, S., Shah, S. K., y Shah, J. M. (2020). Impact of Elearning during COVID-19 pandemic among nursing students and teachers of Nepal. International Journal of Science \& Healthcare Research, 5(3), 68-76. https://ijshr.com/IJSHR_Vol.5_Issue.3_July2020/IJSHR0012.pdf

Viner, R., Russell, S., Croker, H., Packer, J., Ward, J., Stansfield, C., Mytton, O., Bonell, C., y Booy, R. (2020). School closure and management practices during coronavirus outbreaks including COVID-19: a rapid systematic review. The Lancet Child \& Adolescent Health, 4(5), 397-404. https://doi.org/10.1016/S23524642(20)30095-X

Watson, F., Castano, M., y Ferdinand, D. (2017). Instructional Strategies to Help Online Students Learn: Feedback from Online Students. TechTrends, 61(5), 420427. https://doi.org/10.1007/s11528-017-0216-y

Organización Mundial de la Salud [OMS]. (2009, 11 de septiembre). Measures in school settings. Pandemic (H1N1) 2009 briefing note 10. World Health Organization.

https://www.who.int/csr/disease/swineflu/notes/h1n1_school_measures_200909 $11 / \mathrm{en} /$

Contraste de experiencias de estudiantes universitarios en dos escenarios educativos: enseñanza en línea vs. enseñanza remota de emergencia. Niño, Castellanos-Ramírez y Patrón. 